\title{
Flow Field Abstraction and Vortex Detection for MR Velocity Mapping
}

\author{
Yin-Heung Pauline $\mathrm{Ng}^{1}$, Bernardo Silva e Carmo ${ }^{2}$, and Guang-Zhong Yang ${ }^{1}$ \\ ${ }^{1}$ Royal Society/Wolfson Medical Image Computing Laboratory, \\ Imperial College London, London, United Kingdom \\ \{y.h.ng, g.z.yang\} @imperial.ac.uk \\ ${ }^{2}$ Department of Electronics and Computer Science, \\ University of Southampton, SO17 1BJ, United Kingdom \\ bsc01r@ecs.soton.ac.uk
}

\begin{abstract}
The evolution of blood flow patterns (vortical flow features in particular) and their interactions with cardiovascular structure is important in the study of cardiovascular function. The winding index method is widely used for vortex detection and has shown to be very sensitive to noise. We propose a novel technique for the detection of vortical flow features that consists of three main components: flow restoration, abstraction and vortex detection. Firstly, an abstract flow field is obtained by clustering the flow field restored using our proposed restoration method. Afterwards, critical points are computed from the abstract flow field and then phase portrait theory is employed to identify vortices. The proposed technique is applied to 12 sets of MR velocity maps acquired from patients with sequential MR examination following myocardial infarction and the results show that vortical flow patterns are more readily detected compared to the winding index method.
\end{abstract}

\section{Introduction}

The analysis of blood flow patterns and their interaction with cardiovascular structure plays an important role in the study of cardiovascular function. In particular, vortical motion, known to exist in the diastolic phase of the cardiac cycle [1], is the most important flow feature and its evolution over different phases of the cardiac cycle can provide important insight into the health status of the heart. Previous research [2] has shown that in order to achieve a comprehensive and integrated description of flow in health and disease, it is necessary to characterise and model both normal and abnormal flows and their effects. This permits the establishment of links between blood flow patterns and the localised genesis and development of cardiovascular disease.

With the ability to acquire multi-dimensional cine flow data, Magnetic Resonance (MR) velocity imaging is increasingly used for acquiring in vivo flow details. The technique, however, is generally subject to a certain amount of noise and this poses a major problem for automatic quantitative analysis of flow features. To tackle this problem, a vector field restoration method is needed. In recent years, total variation (TV) based restoration method, first introduced by Rudin [3] for restoration of scalar images, has received a lot of interest and several researchers [4][5] have extended the 
method for vector images. We have developed a TV-based variational method [6] for restoring vector field and finding the optimal solution of a regularisation parameter, which is known to affect the restoration result significantly. This restoration scheme has been proven to restore both 2D and 3D flow field effectively [7].

With the recent advances in in vivo MR flow imaging techniques, a large amount of velocity data can be generated rapidly to provide a comprehensive measurement of flow information. Therefore, it is desirable to perform a systematic study and generate a compact topological description of complex flow patterns depicted by MR velocity imaging. Computation and visualisation of vector field topology was first introduced by Helman and Hesselink [8]. In recent years, several methods based on hierarchical clustering have been proposed for vector field abstraction. They can generally be divided into two categories: a top-down strategy and a bottom-up strategy. In both cases, a hierarchical tree is generated to represent the vector field. Heckel et al. [9] used a top-down approach where a discrete vector field is segmented into a disjoint set of clusters, whereas Telea et al. [10] used a bottom-up clustering approach by recursively merging similar clusters to form larger cluster. Lodha et al. [11] adopted the same clustering approach as Telea et al., but with a different error metric to preserve topology of the vector field. More recently, Garcke et al. [12] have proposed a continuous clustering method for simplifying vector fields. This method is based on the Cahn-Hilliard model that describes phase separation and coarsening in binary alloys. The essence of the technique is similar to the other bottom-up methods in that it builds a multi-scale vector field representation by merging neighbouring cells, but it was done in a continuous manner. Generally speaking, all these methods use a single vector to represent a cluster and are well suited for visualisation purposes, however, given a set of such clusters, it is difficult to recover the original vector field.

For the purpose of providing a better ground for vortices detection, we propose to use local linear expansion for representing a cluster of $2 \mathrm{D}$ vectors, with each plane representing a velocity component. By using this representation, the neighbourhood of a critical point can be compactly described. The advantage of using this representation is that given a cluster, the approximation of the original vectors can be easily deduced from the planes. We show that this approach greatly reduces the amount of information needed to describe complex flow fields while preserving all the important critical points intrinsically. Comparison to the traditional approach of vortex detection with winding index is also provided [13][14], and we demonstrate the much-improved sensitivity provided by the proposed new method.

\section{Materials and Methods}

\subsection{Flow Field Restoration}

Flow velocity images acquired by MR velocity-mapping are generally subject to a certain amount of noise that is intrinsic to system hardware setup and those specific to patient movement in relation to imaging sequence designs. To improve the accuracy of the quantitative analysis of the evolution of topological flow features, it is essential to restore the original flow fields. In this study, we formulated a constrained optimisation problem for the restoration of direction $\mathbf{u}$, which is defined as $\mathbf{u}=\mathbf{v} /|\mathbf{v}|$ where $\mathbf{v}$ is the original velocity vector. The numerical scheme of this restoration method [6] 
can be defined as follows. Firstly, the strength function $e(\mathbf{u} ; \quad)$ at voxel can be defined as:

$$
e(\mathbf{u} ; \alpha)=\left[\sum_{\beta \in N_{\alpha}} d_{l}^{2}\left(\mathbf{u}_{\beta}, \mathbf{u}_{\alpha}\right)\right]^{\frac{1}{2}}
$$

where $N_{\alpha}$ denotes the neighbourhood of pixel $\alpha$ and $d_{l}$ denotes the embedded Euclidean distance in $\mathbf{S}^{2}$. The TV-energy of the direction field in domain $\Omega$ is then:

$$
E^{T V}=\sum_{\alpha \in \Omega} e(\mathbf{u} ; \alpha)=\sum_{\alpha \in \Omega}\left[\sum_{\beta \in N_{\alpha}} d_{l}^{2}\left(\mathbf{u}_{\beta}, \mathbf{u}_{\alpha}\right)\right]^{\frac{1}{2}}
$$

and the constrained optimisation can be written as:

$$
\min E^{T V}=\sum_{\alpha \in \Omega}\left[\sum_{\beta \in N_{\alpha}} d_{l}^{2}\left(\mathbf{u}_{\beta}, \mathbf{u}_{\alpha}\right)\right]^{\frac{1}{2}} \text { subject to } h(\mathbf{u})=\frac{1}{2}\left[\sum_{\alpha \in \Omega} d_{l}^{2}\left(\mathbf{u}_{\alpha}, \mathbf{u}_{\alpha}^{0}\right)-|\Omega| \sigma^{2}\right]=0
$$

where $\mathbf{u}^{0}$ denotes the original noisy image. The corresponding Lagrange function, also referred to as the unconstrained TV energy, is:

$$
L(\mathbf{u} ; \lambda)=E^{T V}+\lambda \cdot h(\mathbf{u})=\sum_{\alpha \in \Omega}\left[\sum_{\beta \in N_{\alpha}} d_{l}^{2}\left(\mathbf{u}_{\beta}, \mathbf{u}_{\alpha}\right)\right]^{\frac{1}{2}}+\frac{\lambda}{2}\left[\sum_{\alpha \in \Omega} d_{l}^{2}\left(\mathbf{u}_{\alpha}, \mathbf{u}_{\alpha}^{0}\right)-|\Omega| \sigma^{2}\right]
$$

Note that at the optimal solution, the constraint is met, i.e. $h(\mathbf{u})$ equals zero, and therefore $L(\mathbf{u} ; \quad)$ equals $\mathrm{E}^{\mathrm{TV}}$. The discrete form of the First Order Lagrangian Method can be derived and written as a pair of iterative equations as follows:

$$
\left\{\begin{array}{l}
\mathbf{u}_{\alpha}^{n+1}=\mathbf{u}_{\alpha}^{n}+\Delta t \cdot \prod_{\mathbf{u}_{\alpha}}\left[\sum_{\beta \in N_{\alpha}} w t_{\alpha}^{\beta} \mathbf{u}_{\beta}+\lambda^{n} \mathbf{u}_{\alpha}^{0}\right] \\
\lambda^{n+1}=\lambda^{n}+\Delta t \cdot \frac{1}{2}\left[\sum_{\alpha \in \Omega} d_{l}^{2}\left(\mathbf{u}_{\alpha}, \mathbf{u}_{\alpha}^{0}\right)-|\Omega| \sigma^{2}\right]
\end{array}\right.
$$

And $\prod_{u}$ denotes projection onto the plane orthogonal to $\mathbf{u}$ and $\Delta \mathrm{t}$ denotes the step size.

\subsection{Flow Field Abstraction and Vortical Feature Detection}

To generate an abstract flow field from a dense vector field, we apply a hierarchical clustering algorithm to merge individual flow vectors into clusters through an iterative process. A crucial factor to a good clustering algorithm is the choice of the representation for a cluster of vectors. In our study, local linear expansion of the velocity vectors is employed to represent a cluster of $2 \mathrm{D}$ vectors. This representation is effectively the same as using two planes with limited scopes, one for each velocity component, to locally represent a cluster of vectors. The planes are fitted using the least squares method and the cost of merging two clusters is defined as the second moment of all the points in the merged cluster. 
The choice of using planes to represent a flow field is particularly suitable for regions near critical points, thus, this abstraction technique intrinsically preserves critical points and provides a good foundation for identification of critical points. The first step of the clustering algorithm is to divide the image domain into clusters of $2 \times 2$ vectors. Each cluster is represented by two planes which can be generated using the least squares method. Then, for each neighbouring cluster pair, the associated cost of merging the pair is calculated and stored in a pool. After the initialisation step, the following steps are repeated until all clusters are merged to form one single cluster which contains all individual vectors : i) the pair of clusters with the smallest merging cost in the pool is selected and merged to form a new cluster, and ii) the cost of merging the newly merged cluster with its neighbours is calculated and inserted into the pool. By repeatedly merging clusters, a hierarchical tree is constructed in the process, with each node representing a cluster and its children representing its subclusters. Once the hierarchical tree is constructed, abstract flow field with various compression ratios can then be obtained from this tree efficiently.

The detection of critical points in vector fields require the evaluation of the Jacobean matrix at positions of zero velocity. The traditionally adopted method of winding index is sensitive to image noise. With the proposed flow field abstraction method, this problem is naturally avoided as the Jacobean matrix can be readily retrieved from the velocity planes. After the critical points are extracted, they can be classified by using the phase portrait theory [15] to separate them into attracting/repelling focuses, attracting/repelling nodes, planar vortex, or saddle by solving for the eigenvalues of the Jacobean matrix. For vortices, the associated eigenvalues will have large imaginary values.

\section{Results and Discussion}

To demonstrate the effectiveness of our restoration method, a 3D Computational Fluid Dynamic (CFD) dataset simulating a 3D vortex with added Gaussian noise was used for examining the restoration process. A sample noise-free image, the corresponding noisy image and the restored image are shown in Figure 1. To assess the convergence behaviour of the proposed technique, the constrained and unconstrained energy terms were recorded at each iteration and plotted in Figure 1d. It is found that both energy terms converge to the same value as expected. The rate of convergence depends on the choice of step size, which is typically of the order of 0.01 . The algorithm converged after approximately 200 iterations. A more detailed analysis of the performance of this restoration method can be found in [7].

With the convergence behavior of the restoration process fully analysed, 12 sets of 2D MR flow velocity data acquired from six patients with sequential examination following myocardial infarction is then used for evaluating our proposed flow restoration, abstraction and vortex detection method. All images were acquired using a Marconi whole body MR scanner operating at 1.5T. Cine phase contrast velocity mapping was performed using a field even echo rephrasing (FEER) sequence with a TE of $14 \mathrm{~ms}$. The slice thickness was $10 \mathrm{~mm}$ and the field of view was $30-40 \mathrm{~cm}$.

To analyse the performance of our proposed flow abstraction algorithm, a number of abstract flow fields at various compression ratios are generated for each restored flow field and the abstract flow fields are then used to reconstruct the original flow 
field. The reconstructed flow fields are compared with the original uncompressed flow fields (Figure 2). It is found that, with our proposed plane representation, the salient flow features are well preserved in the abstract flow field even at high compression rate. The results show clear evidence that with a suitable representation, a complex flow field can be closely represented in a much more compact way. For reference purpose, the normalised magnitude error and the angular error at various compression levels are listed in Table 1.

To validate our proposed vortex detection method, all frames acquired during diastole from the 12 datasets were selected. The datasets have a temporal resolution of $40 \mathrm{~ms}-50 \mathrm{~ms}$ and the diastolic phase is covered in about 5-8 frames. A sample sequence of flow images with vortices extracted by using the proposed method and the winding index method are shown alongside in Figure 3. The images clearly show that vortices are more readily detected using our flow abstraction and vortex detection method.

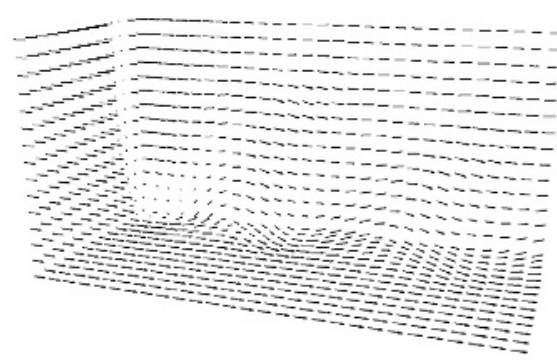

(a)

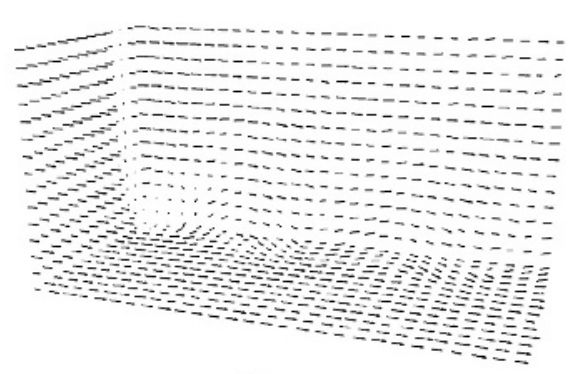

(c)

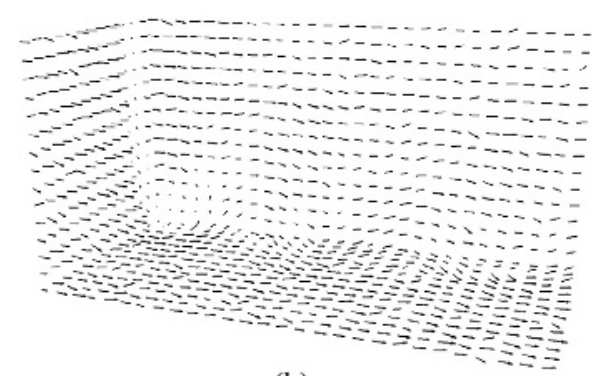

(b)

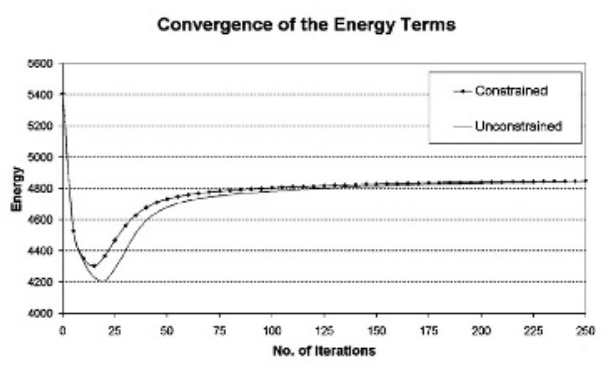

(d)

Fig. 1. Result of our restoration method using a synthetic 3D dataset which simulates flow containing a vortex. (a) Original noise-free flow image. b) With added Gaussian noise. (c) Restored flow image. (d) Convergence plot of the constrained and unconstrained energy terms against the number of iterations for the $3 \mathrm{D}$ synthetic dataset. 


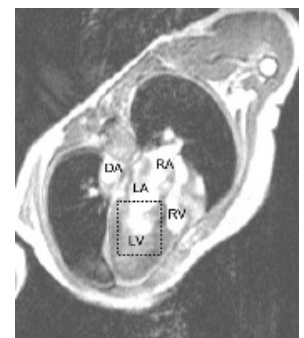

(a) HLA MR image

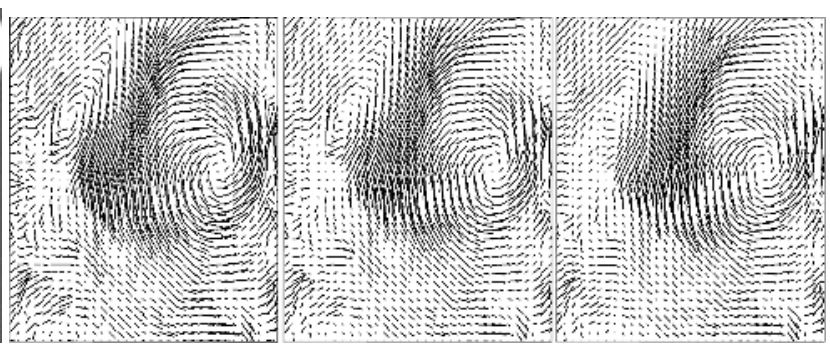

(b) Original

(c) $80 \%$ compression (d) $90 \%$ compression

Fig. 2. (a) A horizontal long axis (HLA) MR image of the left ventricle with the four main chambers and the descending aorta labeled (LA: left atrium; RA: right atrium; LV: left ventricle; RV: right ventricle; DA: descending aorta). (b) The flow pattern of region marked in (a) at the onset of diastole. (c-d) Reconstructed flow field from compact flow field at two different compression levels.

Table 1. Magnitude and angular error of the abstract flow fields depicted in Fig. 2. The original flow field contains 1440 vectors

\begin{tabular}{|c|c|c|c|}
\hline $\begin{array}{c}\text { Compression } \\
\text { Ratio }\end{array}$ & $\begin{array}{c}\text { No. of } \\
\text { Clusters }\end{array}$ & $\begin{array}{c}\text { Angular Error } \\
\text { (degree) }\end{array}$ & $\begin{array}{c}\text { Magnitude Error } \\
\text { (normalised) } \mathbf{x 1 0}^{-3}\end{array}$ \\
\hline \hline $75 \%$ & 360 & 10.3 & 3.5 \\
\hline $80 \%$ & 288 & 11.2 & 4.5 \\
\hline $85 \%$ & 216 & 13.6 & 6.2 \\
\hline $90 \%$ & 144 & 15.3 & 8.6 \\
\hline $95 \%$ & 72 & 25.0 & 12.4 \\
\hline
\end{tabular}

To analyse the sensitivity, defined as the number of correctly identified vortices divided by the number of known vortices, of the two vortex detection methods, the number of major vortices correctly identified by these two methods are recorded and compared against the number of major vortices identified manually. The sensitivities of both methods are plotted in Figure 4. It is found that our proposed method has achieved an average sensitivity of around 90\%, much higher than that of the traditional winding index method. The main drawback of our proposed method is that some unwanted vortices, either minor vortices or false identification, are identified along with the major vortices. This, however, does not pose a major problem to our study as these unwanted vortices can easily be filtered by applying a simple tracking algorithm to locate the major vortices.

\section{Conclusions}

In this study, we proposed a new method for flow field abstraction and vortical flow feature extraction in MR velocity maps. The proposed flow abstraction algorithm can greatly reduce the amount of information needed to describe a complex flow field while preserving all the important critical points. It is shown that the abstract flow field serves as a more reliable ground for critical point detection. 


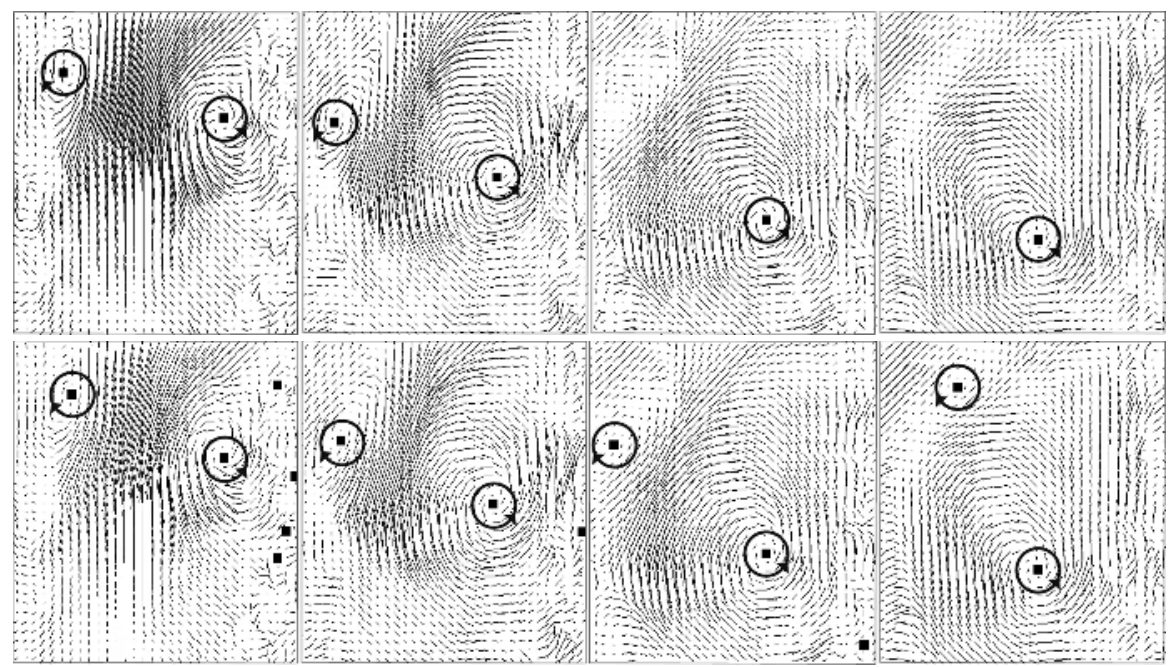

Fig. 3. The flow patterns and the extracted vortices at several stages of the diastolic phase of the cardiac cycle from one of the patients studied. All extracted vortices (including false and minor vortices) are marked with black dots where the major vortices are highlighted with a circle and an arrow to indicate its rotational direction. (Top) The depicted vortices were extracted by the traditional winding index method. (Bottom) The flow patterns were reconstructed from the abstraction method and vortices were then extracted using the new vortex detection method.

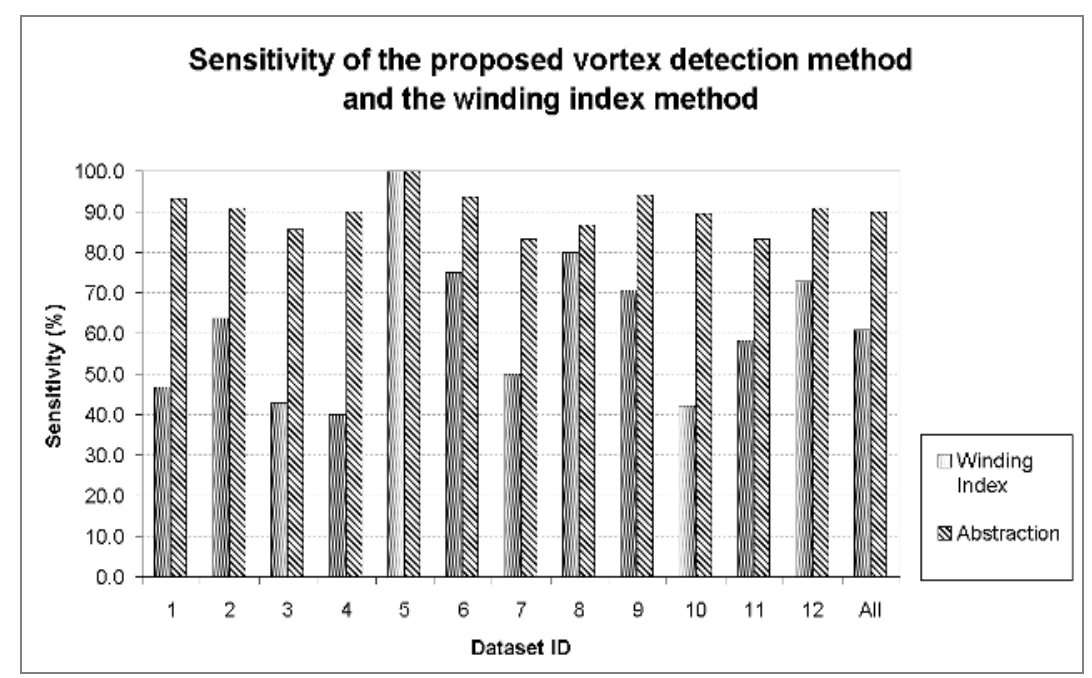

Fig. 4. A comparison of the sensitivity of the proposed vortex detection method and the winding index method using 12 sets of MR flow images acquired from 6 patients at various time following myocardial infarction. It can be seen that the proposed method consistently performed better than the winding index method. 
We have also demonstrated that the proposed flow abstraction algorithm together with the vortex detection algorithm is effective in detecting vortices. Its sensitivity on 12 sets of images is much higher than the winding index method. With the compact representation of flow field and detected vortices, we can analyse and compare the dynamical indices of the blood flow in patients and normal subjects and ultimately establish the links between blood flow patterns and the localised genesis and development of cardiovascular disease.

Acknowledgements. YHP Ng is supported by the Croucher Foundation, Hong Kong. BS Carmo is supported by a Portuguese FCT grant and a UK EPSRC grant.

\section{References}

1. Kim WY, Walker PG, Pedersen EM, Poulsen JK, Oyre S, Houlind K, Yoganathan AP. Left ventricular blood flow patterns in normal subjects: a quantitative analysis by threedimensional magnetic resonance velocity mapping. J. Amer. Coll. Cardiol., 26(1): 224-38, 1995.

2. Yang GZ. The role of quantitative MR velocity imaging in exploring the dynamics of in vivo blood flow. IEEE Engineering in Medicine and Biology, 17(3): 64-72, 1998.

3. Rudin L, Osher S. Total variation based image restoration with free local constraints. Proc. $1^{\text {st }}$ IEEE Int. Conf. Image Processing, 131-35, 1994.

4. Blomgren P, Chan TF. Color TV: total variation methods for restoration of vector valued images. IEEE Trans. Image Processing, 7(3): 304-09, 1998.

5. Chan T, Kang SH, Shen J. Total variation denoising and enhancement of color images based on the CB and HSV color representation. J. Visual Comm. Image Rep., 12(4): 422-35, 2001.

6. Ng YHP, Yang GZ. Vector-valued image restoration with application to magnetic resonance velocity imaging. Journal of WSCG, 11(2): 338-45, 2003.

7. Ng YHP, Carmo BS, Prügel-Bennett A, Yang GZ. A first-order Lagrangian-based variational approach for 3D flow vector field restoration. Proc. CARS 2003, Int. Congress Series 1256: 1185-90, 2003.

8. Helman JL and Hesselink L. Visualizing vector field topology in fluid flows. IEEE Computer Graphics \& Applications, 11(3): 36-46, 1991.

9. Heckel B, Weber G, Hamann B, Joy KI. Construction of vector field hierarchies. Proc. IEEE Visualization, 19-25, 1999.

10. Telea A, van Wijk JJ. Simplified representation of vector fields. Proc. IEEE Visualization, 35-42, 1999.

11. Lodha SK, Renteria JC, Roskin KM. Topology preserving compression of 2D vector fields. Proc. IEEE Visualization, 343-50, 2000.

12. Garcke H, Preußer T, Rumpf M, Telea A, Weikard U, van Wijk JJ. A phase field model for continuous clustering on vector fields. IEEE Transactions on Visualization and Computer Graphics, 7(3): 230-41, 2001.

13. Ford RM, Strickland RN, Thomas BA. Image models for 2-D flow visualization and compression. CVGIP:Graphical Model and Image Processing, 56(1): 75-93, 1994.

14. Yang GZ, Mohiaddin RH, Kilner PJ, Firmin DN. Vortical flow feature recognition: a topological study of in vivo flow patterns using MR velocity mapping. J. Comp. Assist. Tomography, 22(4): 577-86, 1998.

15. Rao R, Jain R. Analysing oriented textures through phase portraits. Proc. 10th Int. Conf. Pattern Recognition, 336-40, 1990. 